\title{
On the Design of Slot Cut Circularly Polarized Circular Microstrip Antennas
}

\author{
Amit A. Deshmukh, Saleha A. Shaikh, Ami A. Desai, Kshitij A. Lele, Sudesh Agrawal \\ Department of Electronics and Telecommunication, Dwarkadas J. Sanghvi College of Engineering (DJSCE), \\ Mumbai, India \\ Email: amitdeshmukh76@rediffmail.com, salehaakbar91@gmail.com
}

Received 9 November 2015; accepted 25 January 2016; published 28 January 2016

Copyright (C) 2016 by authors and Scientific Research Publishing Inc.

This work is licensed under the Creative Commons Attribution International License (CC BY).

http://creativecommons.org/licenses/by/4.0/

(c) (i) Open Access

\begin{abstract}
Circularly polarized microstrip antenna is frequently realized by cutting the slot inside the patch and feeding it along the diagonal axis. In the reported literature, procedure to design them at any given frequency is not available. In proposed work, circularly polarized slot cut circular microstrip antenna at $900 \mathrm{MHz}$ is discussed. By studying the surface current distributions at two orthogonal modes, formulations in their resonant length are proposed. The frequencies calculated using them closely agree with simulated results. Using proposed formulation, procedure to design circular polarized antennas at different frequencies is presented that gives circular polarized response. Thus, proposed work will be helpful to design similar circular polarized circular microstrip antenna at any desired frequency.
\end{abstract}

\section{Keywords}

Circular Microstrip Antenna, Circular Polarization, Rectangular Slot, Resonant Length Formulation

\section{Introduction}

The circular polarized (CP) microstrip antenna (MSA) is realized either by cutting narrow slot inside the coaxially fed MSA or by using dual feeds in conjunction with the power divider network, or by cutting asymmetrical U-slot or rectangular slot at an appropriate position inside the patch [1]-[11]. Amongst these techniques, U-slot or rectangular slot cut MSAs yields optimum response in terms of gain and axial ratio (AR) bandwidth (BW) [9]-[11]. However in these reported CP MSAs, formulation in resonant length at orthogonal modes and procedure to design them at any other frequency is not explained. Therefore, optimization process to design CP MSAs involves parametric study on different patch parameters using simulation software, followed by experimental validation. In this paper, first rectangular slot cut circular MSA (CMSA) to give CP response in $900 \mathrm{MHz}$ fre- 
quency range, is discussed. On glass epoxy substrate $\left(\varepsilon_{\mathrm{r}}=4.3, \mathrm{~h}=0.16 \mathrm{~cm}, \tan \delta=0.02\right)$, it gives VSWR and AR BW of 20 and $7 \mathrm{MHz}$, respectively. To increase its gain, three layer suspended configuration is proposed which gives VSWR and AR BW of 32 and $9 \mathrm{MHz}$, respectively, with a gain of more than $3 \mathrm{dBi}$. Further by studying surface current distributions at orthogonal modes, formulation in their resonant length in terms of slot and patch dimensions for non-suspended and suspended MSAs, is proposed. The frequencies calculated using them closely agree with the simulated results. Using proposed formulations, procedure to design slot cut CP CMSA at any other frequency in non-suspended and suspended configuration is presented. It gives CP response with formation of small loop inside VSWR $=2$ circle and with the broadside radiation pattern that shows co and cross polarization levels within $3 \mathrm{~dB}$ in $\mathrm{E}$ and $\mathrm{H}$-planes. Thus, proposed formulations can be used to realize $\mathrm{CP}$ response at any desired frequency. All these MSAs were first designed and optimized using IE3D software [12]. For only the optimized and re-designed configurations, experiments were carried out to validate the simulated and proposed design procedure. The MSAs were fed using SMA panel type connector of $0.12 \mathrm{~cm}$ inner wire diameter. The impedance measurements were carried out using R \& S vector network analyzer (ZVH-8). In simulations, infinite ground plane with infinite extent dielectric was assumed. This helps in realizing the simulation of MSA in reduced time. To realize this effect in measurements, size of substrate is taken to be more than six times the substrate thickness in all the direction with respect to patch periphery. The radiation pattern and the co-polar gain were measured using RF source (SMB 100A) and spectrum network analyzer (FSC6). The measurement was carried out in antenna lab wherein required minimum far field distance was kept between reference antenna and antenna under test.

\section{Slot Cut CP CMSA}

The rectangular slot cut CMSA is shown in Figure 1(a). The units of the dimensions and frequencies referred in text and in figures are in $\mathrm{cm}$ and $\mathrm{MHz}$, respectively. The CMSA radius "a" is calculated such that its $\mathrm{TM}_{11}$ mode frequency is $900 \mathrm{MHz}$ [1]. To excite orthogonal modes, slot is cut in the patch center and coaxial feed is placed along the diagonal axis. The parametric study is carried out for length "l" $=0.6\left(0.02 \lambda_{0}\right)$ and for increasing slot width $(w)$. For two orthogonal modes, their frequencies and ratio plots against respective VSWR and AR BWs at each of slot width (w) are shown in Figure 1(b). The surface current distributions for "l" = 0.6 and $w=1.5$ at two modes is shown in Figure 2(a) and Figure 2(b). The slot has degenerated $\mathrm{TM}_{11}$ mode into two orthogonal modes. At each mode, one half wavelength variation in surface currents is present along $\mathrm{x}$ and $\mathrm{y}$-axis. The reduction in the frequency of first mode $\left(\mathrm{f}_{1}\right)$ is higher as slot width is orthogonal to its surface currents. The variation in second mode $\left(\mathrm{f}_{2}\right)$ frequency is marginal. Thus " $\mathrm{f}_{2} / \mathrm{f}_{1}$ " ratio increases with increase in " $w$ ". The optimum result is obtained for $w=1.5 \mathrm{~cm}$ to give simulated VSWR and AR BW of 29 and $8 \mathrm{MHz}$, respectively, as shown in Figure 2(c) and Figure 2(d). The measured BW is $32 \mathrm{MHz}$. For $w>1.5 \mathrm{~cm}, \mathrm{f}_{2} / \mathrm{f}_{1}$ ratio increases that do not maintain the required phase quadrature between two modes, which reduces AR BW.

As the slot cut CP CMSA is optimized on lossy substrate, the gain is less than $0 \mathrm{dBi}$. To improve the same, its three layer suspended configuration is proposed as shown in Figure 3(a) and Figure 3(b). The two layers of glass substrate are separated by an air gap of thickness $0.16 \mathrm{~cm}$, thereby giving total substrate thickness of 0.48 $\mathrm{cm}$. The patch is fabricated on top glass epoxy layer whereas SMA connector is connected to the bottom glass epoxy layer which acts as a ground plane. In suspended configuration, effective dielectric constant $\left(\varepsilon_{r e}\right)$ reduces, that increases patch radius for $f_{\mathrm{TM} 11}=900 \mathrm{MHz}$. The $\varepsilon_{r e}$ is calculated by using Equation (1) and further by using Equations (2) and (3), patch radius is calculated [1]. For $900 \mathrm{MHz}$, radius was found to be $6.5 \mathrm{~cm}$. For suspended

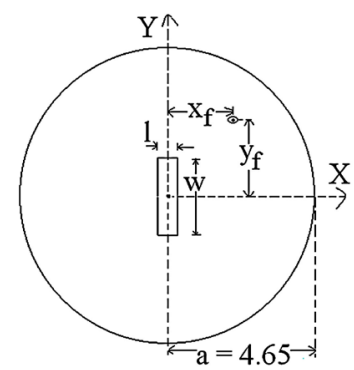

(a)

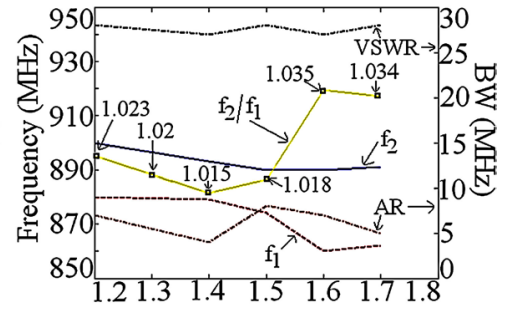

(b) $w(\mathrm{~cm})$

Figure 1. (a) Slot cut CP CMSA; its (b) dual frequency, ratio, AR and VSWR BW plots. 


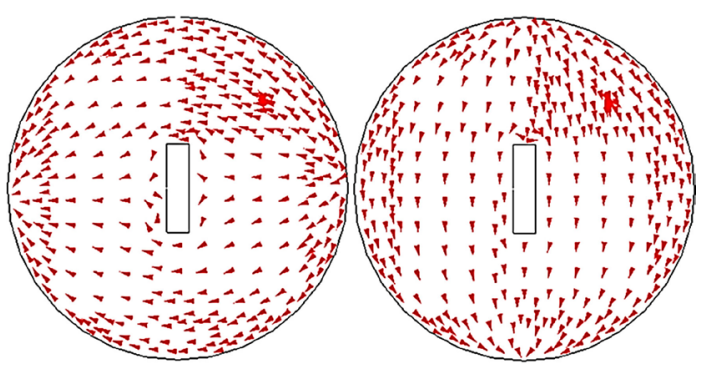

(a) $\mathrm{f}_{1}=874 \mathrm{MHz}$

(b) $\mathrm{f}_{2}=890 \mathrm{MHz}$

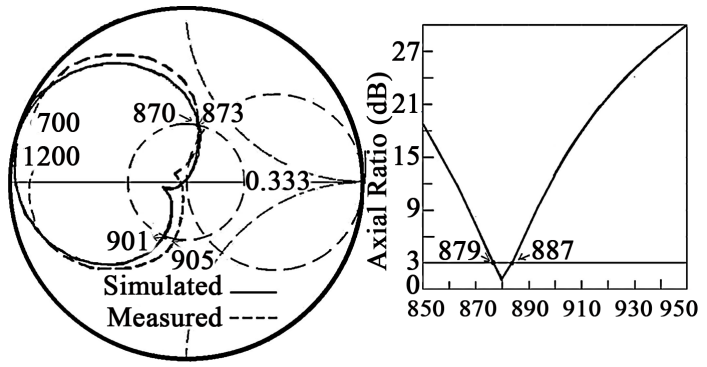

(c)

(d) Frequency (MHz).

Figure 2. (a), (b) Surface current distribution at orthogonal modes and (c) input impedance and (b) simulated AR plots for slot cut CP CMSA.

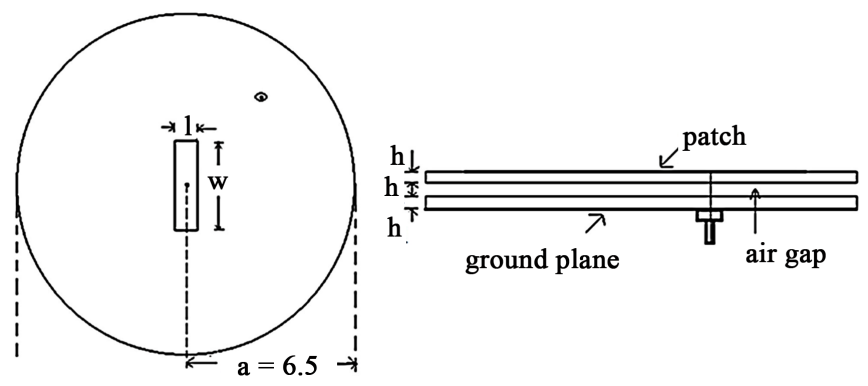

(a)

(b)

Figure 3. (a) Top and (b) side views of suspended slot cut CMSA.

slot cut CMSA, parametric study for variation in slot width is carried out and plots of orthogonal mode frequencies, their ratio, VSWR and AR BW's, are shown in Figure 4.

$$
\begin{gathered}
\varepsilon_{r e}=\frac{3 \varepsilon_{r}}{\varepsilon_{r}+2} \\
k=\frac{8.794}{f_{r} \sqrt{\varepsilon_{r e}}} \\
a=\frac{k}{\sqrt{1+\frac{2 h}{\pi \varepsilon_{r e} k}\left(\ln \left(\frac{\pi k}{2 h}\right)+1.7726\right)}}
\end{gathered}
$$

where,

$h=$ glass epoxy substrate and air gap thickness, $f_{r}=$ resonance frequency in $\mathrm{GHz}$,

$k=$ unit less number, $a$ = patch radius in $\mathrm{cm}, \varepsilon_{r}=$ substrate dielectric constant, $\varepsilon_{r e}=$ effective dielectric constant.

The optimum AR BW is obtained for $w=2.8$ and results for the same are shown in Figure 5(a) and Figure 5(b). The simulated VSWR and AR BW's are 34 and $10 \mathrm{MHz}$, respectively. The suspended slot cut CP CMSA 


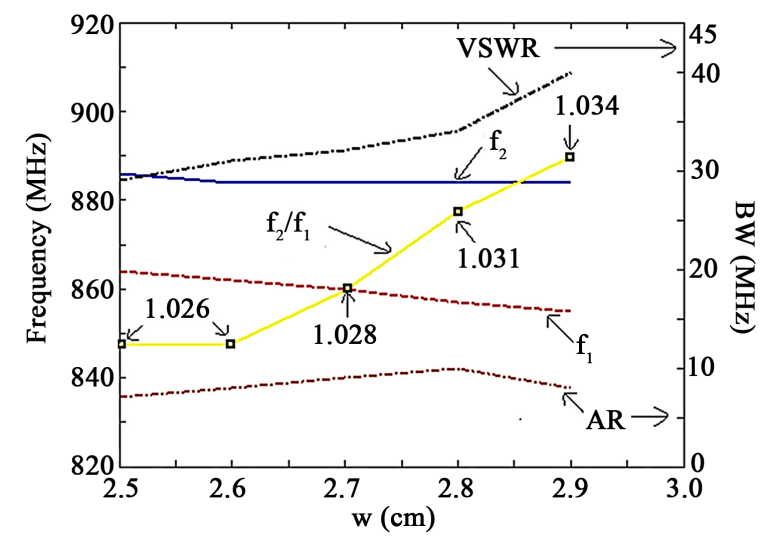

Figure 4. Dual frequencies, their ratio, VSWR and AR BW plots for suspended slot cut CP CMSA.

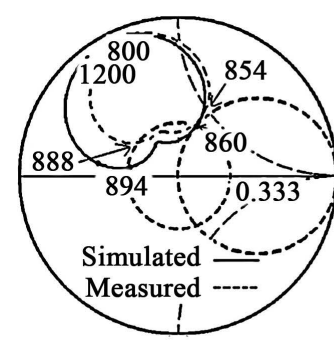

(a)

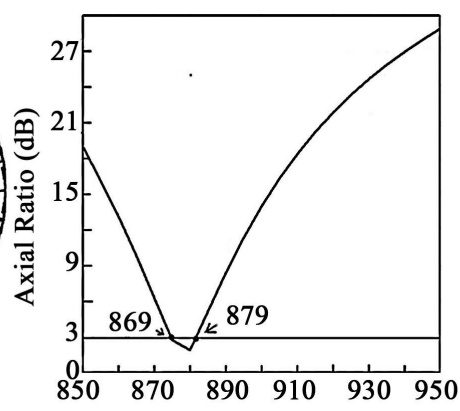

(b) Frequency (MHz)

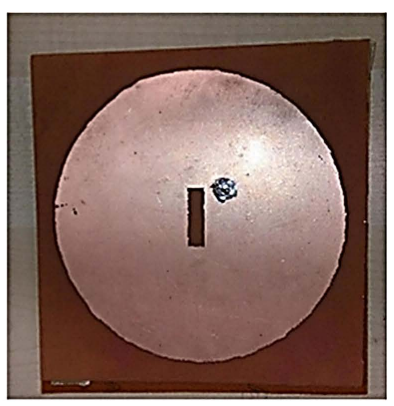

(c)

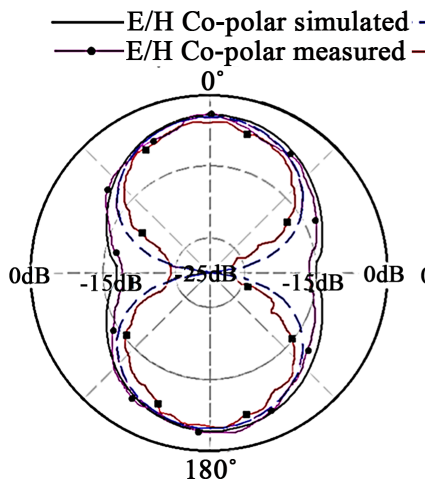

(d) $\mathrm{E}, \mathrm{f}=875 \mathrm{MHz}$

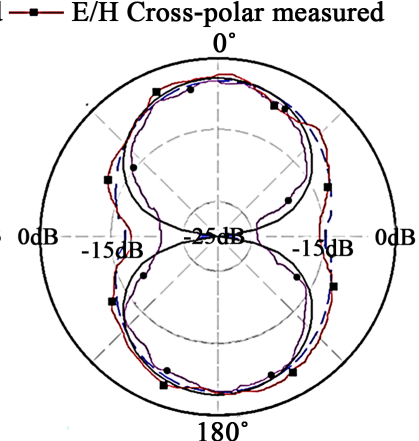

(e) $\mathrm{H}, \mathrm{f}=875 \mathrm{MHz}$

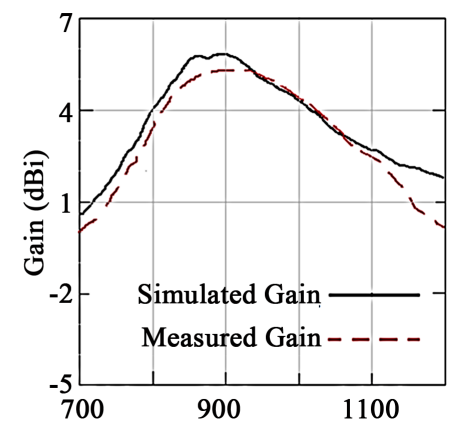

(f) Frequency (MHz)

Figure 5. (a) Input impedance and (b) simulated AR plots, (c) fabricated prototype, (d), (e) radiation pattern and (f) gain variation over BW for suspended slot cut CP CMSA.

was fabricated and experiment was carried out. The measured BW is $32 \mathrm{MHz}$. The fabricated prototype of the configuration is shown in Figure 5(c). The surface current distribution at dual modes in suspended CMSA is similar to that present in non-suspended CMSA. The simulated and measured radiation pattern at center frequency of AR BW and gain variation over VSWR and AR BW's is shown in Figures 5(d)-(f). The co and cross polar levels are within $3 \mathrm{~dB}$ in the broadside direction which indicates the presence of CP. Due to use of suspended configuration antenna gain is more than $3 \mathrm{dBi}$. A good agreement is obtained between simulated and measured results. Although slot cut CP CMSAs discussed above are widely reported but the formulation to design them at any given frequency is not available. Hence most of the available work is based upon the parametric optimization. Here, by studying the surface current distributions, the formulation in resonant length at two orthogonal modes in slot cut CP CMSA is proposed. Further using proposed formulations, procedure to design slot cut CP CMSA at any other frequency is outlined. 


\section{Formulations for Slot Cut CP CMSAS}

In slot cut CMSA, surface currents at two modes are circulating around the slot length. At first mode, they are perturbed in their length due to slot width whereas at second mode, current length is modified due to slot length. The formulation in resonant length on glass epoxy substrate is obtained by modifying patch radius in terms of slot width or length as given in Equations (4) \& (5). The frequencies at two modes are calculated by using resonance frequency equation for $\mathrm{TM}_{11}$ mode as given in Equations (6) and (7). Using Equation (8), \% error between calculated and simulated values is obtained. The frequencies and \% error plots are shown in Figure 6(a). At both the modes, closer agreement is obtained between simulated and calculated values. The formulation in resonant length for suspended CMSA as shown in Figure 3(a) and Figure 3(b), is obtained by modifying patch radius in terms of slot dimensions as given in Equations (9) and (10). The frequencies and \% error are calculated by using Equations (6)-(8) and they are plotted in Figure 6(b). Over the entire range of slot dimension, closer agreement between simulated and calculated values is obtained.

$$
\begin{gathered}
a_{e 1}=a+\frac{w}{4} \\
a_{e 2}=a+\frac{l}{4} \\
f_{r 1}=\frac{1.84118 c}{2 a_{e 1} \pi \sqrt{\varepsilon_{r}}} \\
f_{r 2}=\frac{1.84118 c}{2 a_{e 2} \pi \sqrt{\varepsilon_{r}}} \\
E=\left(\frac{f_{s 1}-f_{r 1}}{f_{s 1}}\right) \times 100
\end{gathered}
$$

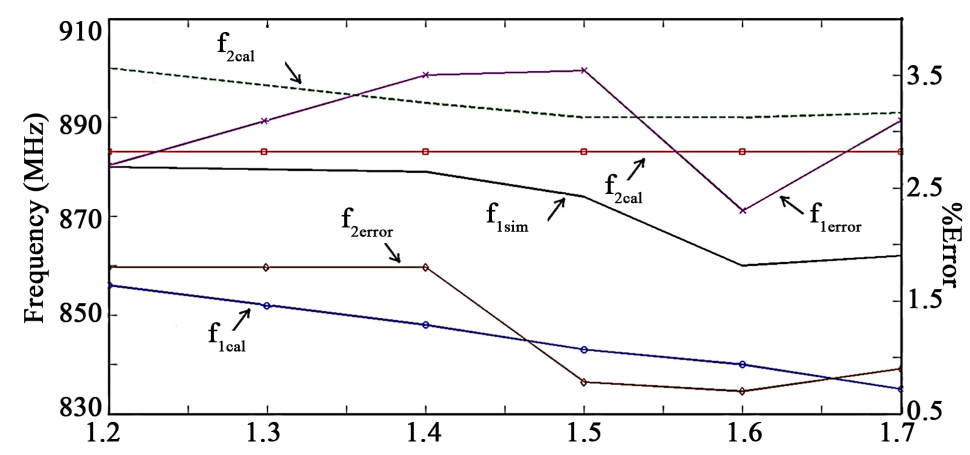

(a) Width (cm)

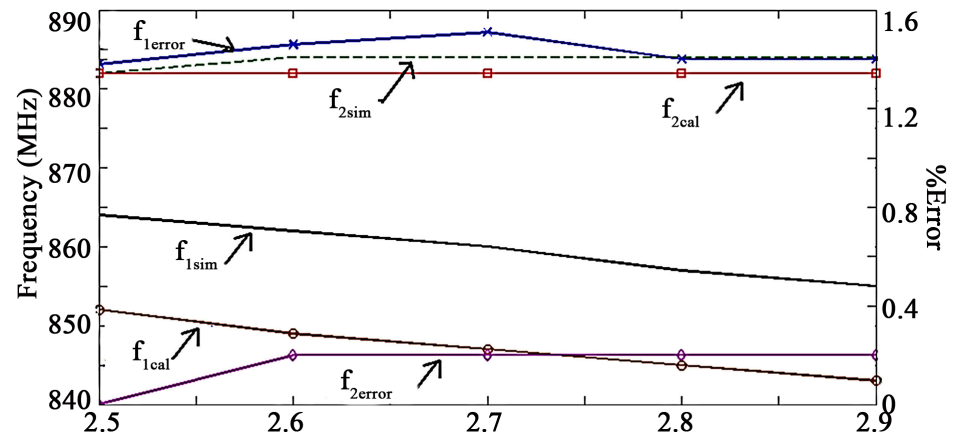

(b) Width (cm)

Figure 6. Dual frequency and \% error plots for (a) non-suspended and (b) suspended slot cut CP CMSAs. 


$$
\begin{aligned}
& a_{e 1}=a+\frac{w}{6} \\
& a_{e 2}=a+\frac{l}{6}
\end{aligned}
$$

The substrate thickness at $900 \mathrm{MHz}$ for non-suspended and suspended configuration is $0.005 \lambda_{0}$ and $0.014 \lambda_{0}$, respectively. Since difference in substrate thickness in two configurations is not larger, their resonant length formulations are nearly identical. As seen from Figure 6(a) and Figure 6(b), calculated frequencies show 2\% to $3 \%$ error with respect to simulated frequencies. As seen from Figure 1(b) and Figure 4, in optimized slot cut CP CMSAs, simulated $\mathrm{f}_{2} / \mathrm{f}_{1}$ ratio is 1.02 and 1.03 for non-suspended and suspended MSAs, respectively. The plots of simulated and calculated dual frequencies and their ratios are shown in Figure 7(a) and Figure 7(b). For the optimized slot width, calculated $\mathrm{f}_{2} / \mathrm{f}_{1}$ ratio nearly equals 1.05 and 1.045 in non-suspended and suspended CMSAs, respectively. Using the proposed formulations, procedure to design slot cut CP CMSAs at different frequencies is presented below.

\section{Design Procedure for Slot Cut CP CMSAS}

In slot cut CP CMSAs at $900 \mathrm{MHz}$, slot length (l) is $0.02 \lambda_{0}$ whereas feed point is placed along the diagonal axis, i.e. at $x_{f}=y_{f}=0.06 \lambda_{0}$. Using proposed formulations, design of slot cut CP CMSA is presented for equivalent patch $\mathrm{TM}_{11}$ mode frequency of 700, 1500, 2000 and $3000 \mathrm{MHz}$, on glass epoxy substrate. The patch radius is calculated by using equations (2) and (3) and at respective frequencies it is found to be, 6.0, 2.8, 2.05 and 1.33 $\mathrm{cm}$. The plots of orthogonal mode frequencies and their ratio are generated by using proposed formulations for $1=$ $0.02 \lambda_{0}$ and increasing slot width, and they are shown in Figures 8(a)-(d). From these plots, value of slot width that gives $\mathrm{f}_{2} / \mathrm{f}_{1}$ of approximately 1.05 , is selected. At 700, 1500, 2000 and $3000 \mathrm{MHz}$, slot width is found to be $2.0,1.0,0.7$ and $0.6 \mathrm{~cm}$, respectively.

At respective frequencies, slot of selected width and $0.02 \lambda_{0}$ in length is cut in the patch center with feed is placed at $\mathrm{x}_{\mathrm{f}}=\mathrm{y}_{\mathrm{f}}=0.06 \lambda_{0}$, along the diagonal axis. The slot cut CMSAs are simulated and their input impedance plots are shown in Figure 9(a) and Figure 9(b) and Figure 10(a) and Figure 10(b).

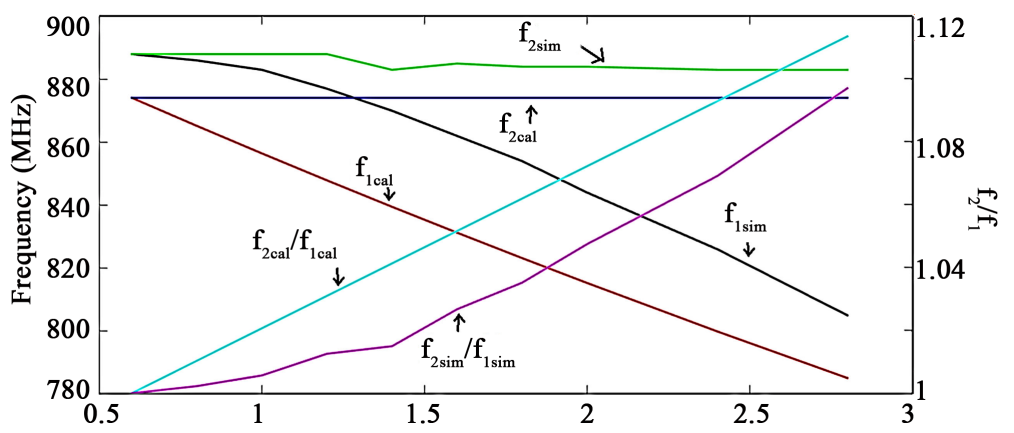

(a) width (cm)

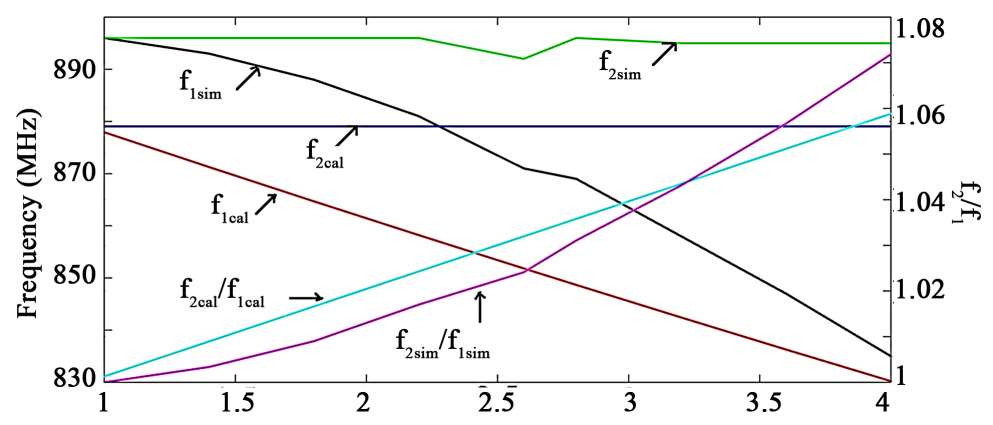

(b) width (cm)

Figure 7. Simulated and calculated dual frequencies and ratio plots for (a) non-suspended and (b) suspended CP CMSAs. 


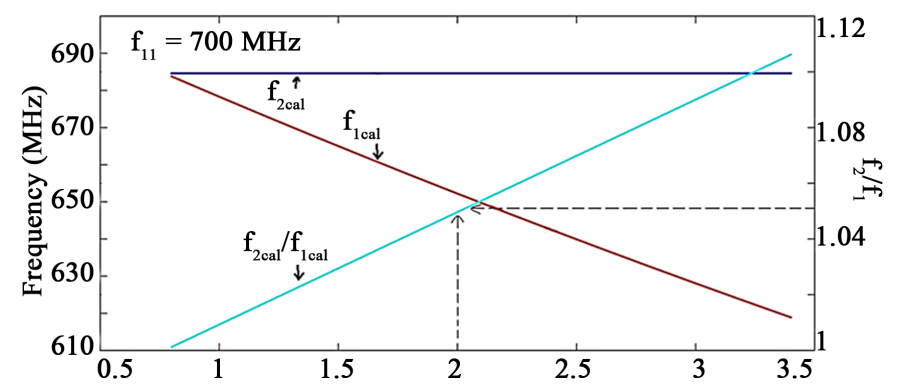

(a) width (cm)

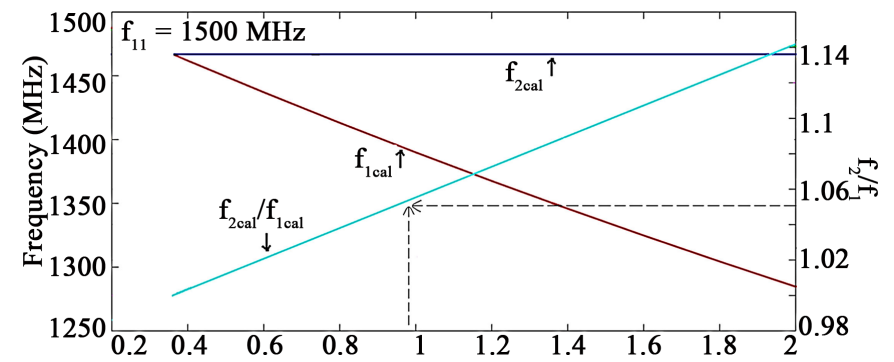

(b) width (cm)

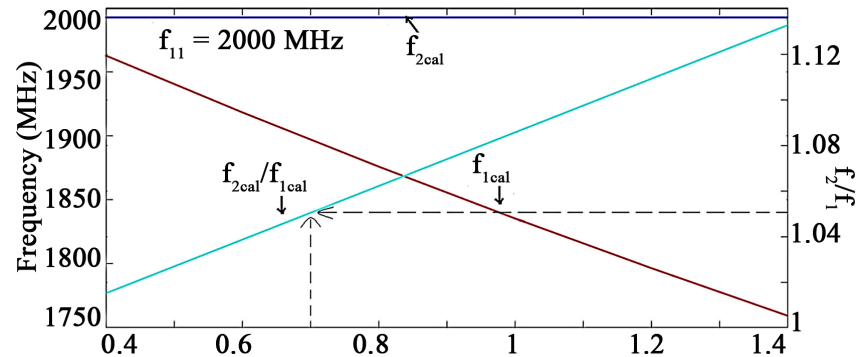

(c) width $(\mathrm{cm})$

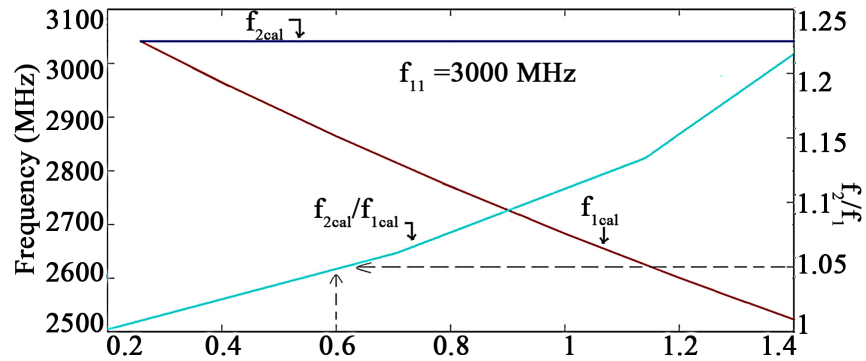

(d) width (cm)

Figure 8. (a)-(d) Dual frequencies and their ratio plots for slot cut CMSA at different frequencies.

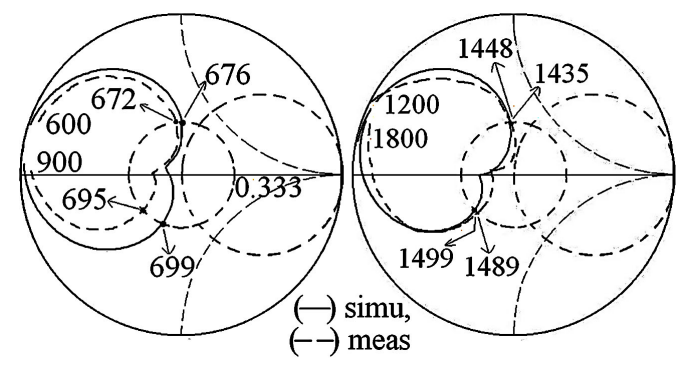

$\begin{array}{ll}\text { (a) } \mathrm{f}_{11}=700 \mathrm{MHz} & \text { (b) } \mathrm{f}_{11}=1500 \mathrm{MHz}\end{array}$

Figure 9. Input impedance plots at (a) 700 and (b) $1500 \mathrm{MHz}$ for slot cut CP CMSA. 


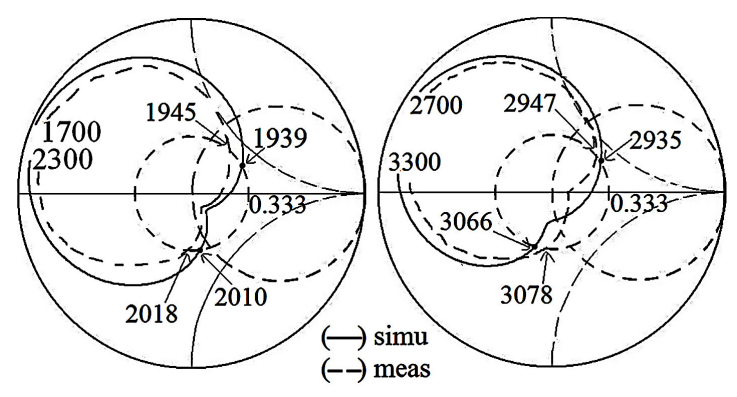

(a) $\mathrm{f}_{11}=2000 \mathrm{MHz}$

(b) $\mathrm{f}_{11}=3000 \mathrm{MHz}$

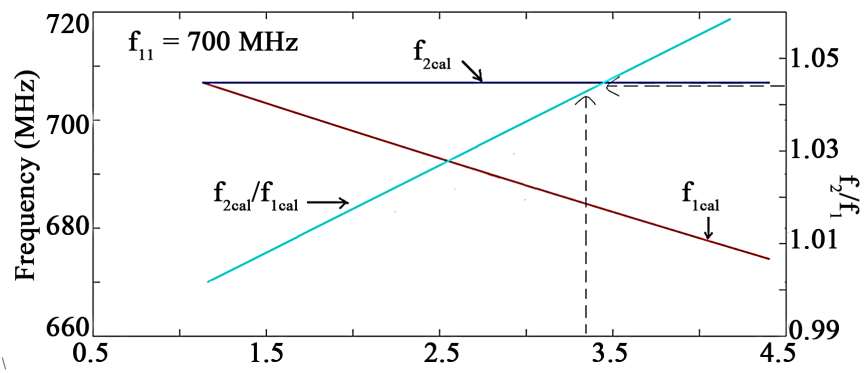

(c) width (cm)

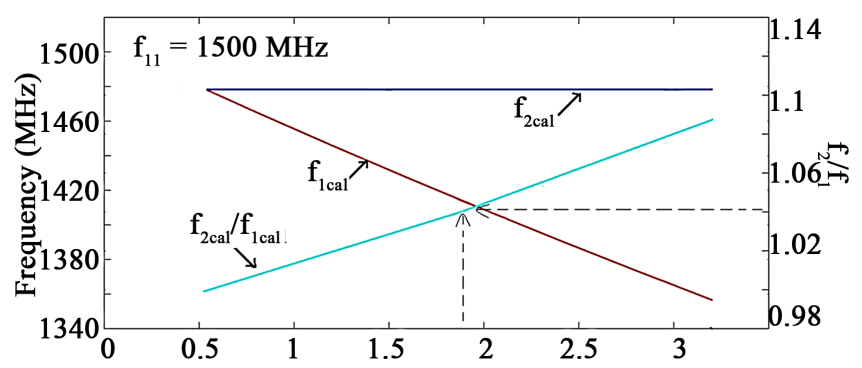

(d) width (cm)

Figure 10. (a), (b) Input impedance plots at two frequencies for slot cut CP CMSA and (c), (d) calculated dual frequencies and ratio plots for suspended slot cut CP CMSAs.

At all the frequencies CP response with formation of small loop (kink) inside VSWR $=2$ circle is obtained. The simulated VSWR and AR BW at 700 and $1500 \mathrm{MHz}$ are, 23 and $6 \mathrm{MHz}$ and 54 and $13 \mathrm{MHz}$, respectively. The simulated VSWR and AR BW's at 2000 and $3000 \mathrm{MHz}$ are, 71 and $18 \mathrm{MHz}$ and 131 and $26 \mathrm{MHz}$, respectively. The measured BW at 700, 1500, 2000 and $3000 \mathrm{MHz}$ are, 23, 51, 73 and $131 \mathrm{MHz}$, respectively. At each of the frequencies, simulated and measured radiation pattern, in the broadside direction shows co and cross polarization levels within $3 \mathrm{~dB}$, which indicates the presence of CP. Further suspended slot cut CP CMSAs are designed. To realize impedance matching for coaxially fed suspended MSAs, at each of the frequencies, the effective substrate thickness is selected to be $\leq 0.03 \lambda_{0}$. Thus at 700,1500 and $2500 \mathrm{MHz}$, three layer suspended configuration with an air gap of $0.16 \mathrm{~cm}$ between two glass epoxy layer is selected. At $3000 \mathrm{MHz}$, two layer suspended configuration in which glass epoxy layer suspended above the ground plane is used. The patch radius is calculated by using Equations (1)-(3) and it is found to be, 8.3, 3.6, 2.6 and $1.9 \mathrm{~cm}$ at 700, 1500, 2000 and 3000 $\mathrm{MHz}$, respectively. Using proposed formulations for suspended CP CMSAs, plots of orthogonal frequencies and their ratio are generated for $\mathrm{l}=0.02 \lambda_{0}$ and increasing slot width. At each of the frequencies they are shown in Figure 10(c) and Figure 10(d) and Figure 11(a) and Figure 11(b).

The value of slot width that gives $\mathrm{f}_{2} / \mathrm{f}_{1}$ of nearly 1.045 is selected as shown in their respective plots. At 700 , 1500, 2000 and $3000 \mathrm{MHz}$, slot width was found to be, 3.4, 1.8, 1.6 and $1.1 \mathrm{~cm}$, respectively. The slot of $0.02 \lambda_{0}$ in length (l) and selected slot width is cut inside suspended CMSAs. The simulated and measured input impedance plots for slot cut CMSAs are shown in Figures 12(a)-(d). At all the frequencies, small loop is observed inside VSWR $=2$ circle in the smith chart. The simulated VSWR and AR BW at 700 and $1500 \mathrm{MHz}$ are, 21 and 8 


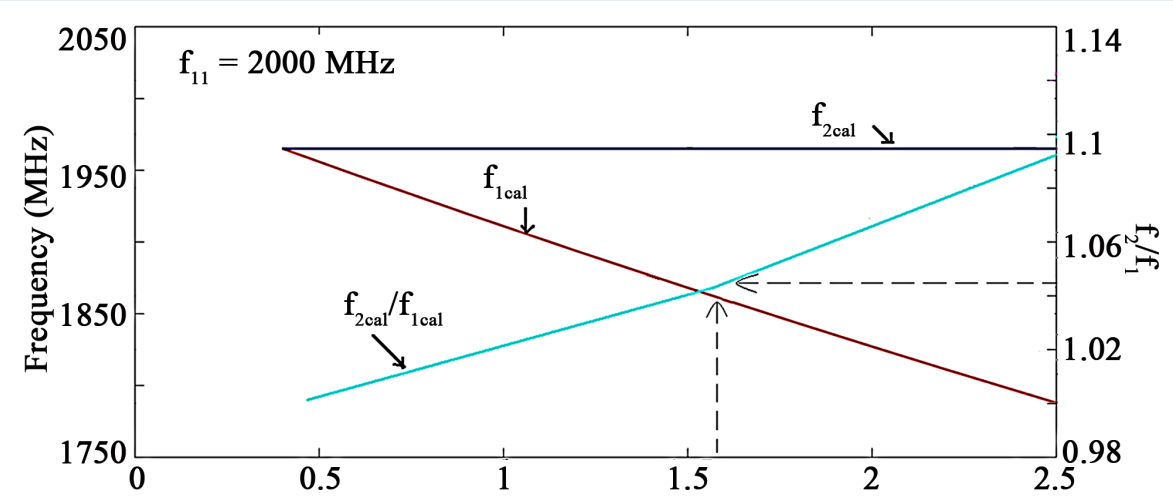

(a) width (cm)

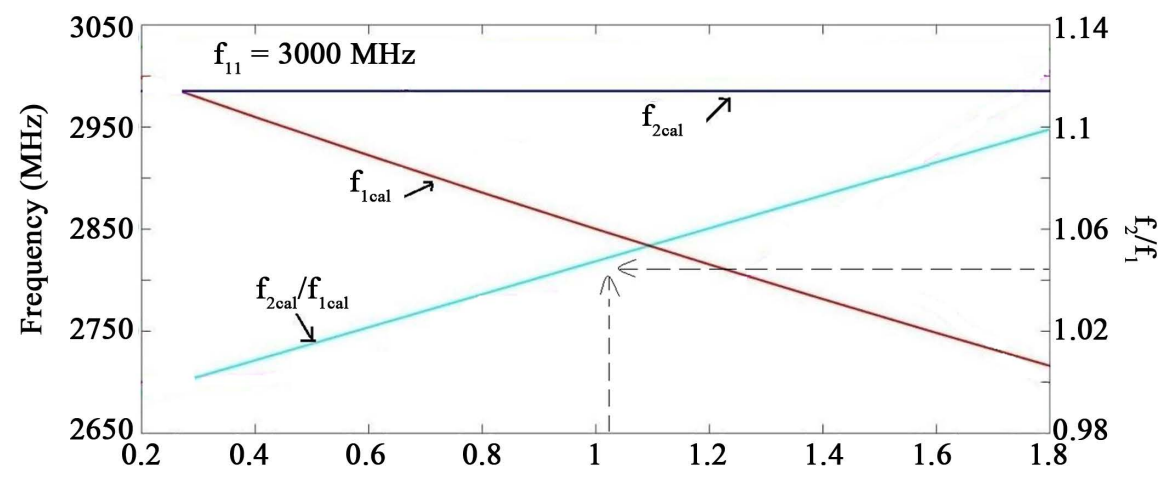

(b) width (cm)

Figure 11. (a), (b) Calculated dual frequencies and their ratio plots for suspended slot cut CP CMSAs at different TM11 mode frequencies.

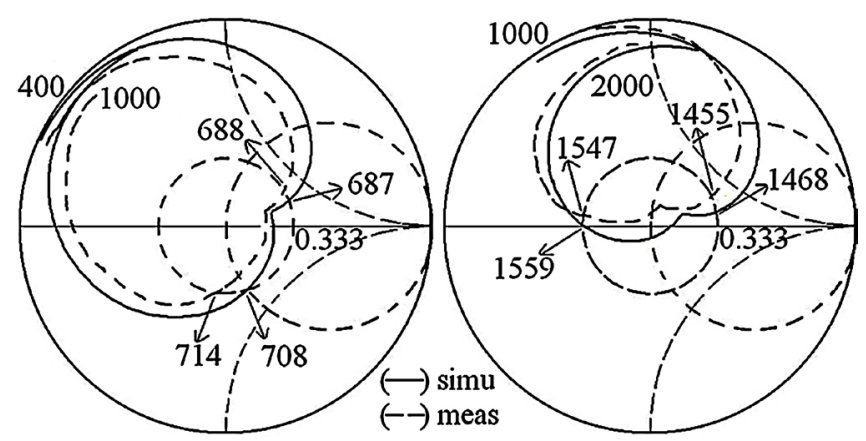

(a) $\mathrm{f}_{11}=700 \mathrm{MHz}$

(b) $\mathrm{f}_{11}=1500 \mathrm{MHz}$

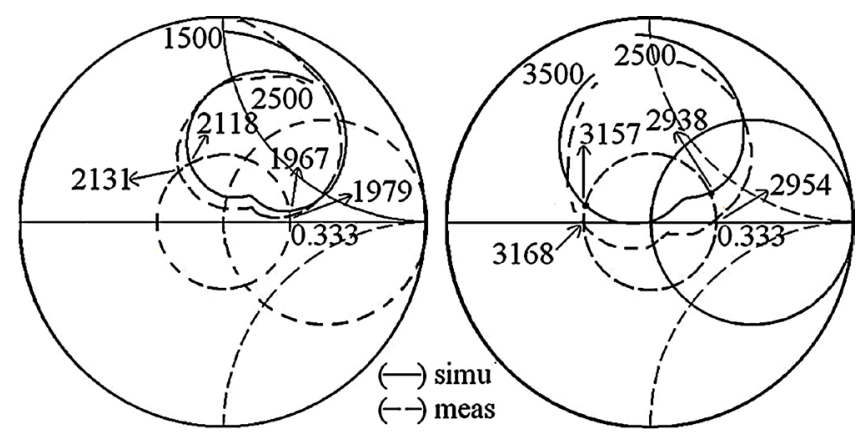

(c) $\mathrm{f}_{11}=2000 \mathrm{MHz}$

(d) $\mathrm{f}_{11}=3000 \mathrm{MHz}$

Figure 12. (a)-(d) Input impedance plots for suspended slot cut CP CMSAs at different TM11 mode frequencies. 
MHz and 91 and 17 MHz, respectively. The simulated VSWR and AR BW's at 2000 and $3000 \mathrm{MHz}$ are, 151 and $18 \mathrm{MHz}$ and 219 and $26 \mathrm{MHz}$, respectively. The measured BW at 700, 1500, 2000 and $3000 \mathrm{MHz}$ are, 26, 92, 152 and $214 \mathrm{MHz}$, respectively. The simulated and measured pattern plots at center frequency of AR BW for suspended CP CMSAs at 1500 and $3000 \mathrm{MHz}$ is shown in Figures 13 (a)-(d). The pattern shows co and cross levels within $3 \mathrm{~dB}$, which indicates the presence of CP. For $\mathrm{f}_{\mathrm{TM} 11}=900 \mathrm{MHz}$, using the proposed formulations slot cut CP CMSA is also designed on RT-duroid substrate $\left(\varepsilon_{r}=2.33, \mathrm{~h}=0.16 \mathrm{~cm}, \tan \delta=0.001\right)$. The patch radius for non-suspended and suspended configurations was calculated to be 6.5 and $7.5 \mathrm{~cm}$, respectively. Using proposed formulations, plots for dual frequencies and their ratio are generated as shown in Figure 14(a) and Figure 14(b). For frequency ratio of 1.05 and 1.045 for non-suspended and suspended CMSAs, slot width was found to be 1.5 and $2.4 \mathrm{~cm}$, respectively. The slot of $0.02 \lambda_{0}$ in length and selected slot width is cut inside the circular patch. The simulated and measured input impedance plots are shown in Figure 15(a) and Figure 15(b).

For non-suspended CMSA, simulated VSWR and AR BWs are, 13 and $5 \mathrm{MHz}$, respectively. The measured BW is $12 \mathrm{MHz}$. For suspended CMSAs simulated values are, 26 and $4 \mathrm{MHz}$, respectively whereas measured $\mathrm{BW}$ is $24 \mathrm{MHz}$. The pattern shows difference between co and cross polarization levels to be less than $3 \mathrm{~dB}$ in the broadside direction. Thus proposed work will be useful for designing slot cut CP CMSAs at any desired frequency and on different substrates.

\section{Conclusion}

The design of narrow slot cut CP CMSA at $900 \mathrm{MHz}$ is discussed. The slot degenerates patch $\mathrm{TM}_{11}$ mode into two orthogonal modes, to realize CP response. To improve the gain, three layer suspended design of slot cut CP CMSA is also proposed which gives gain of more than $3 \mathrm{dBi}$. In the reported literature on slot cut CP CMSAs, design guidelines to realize similar CP antennas at any given frequency are not available. Therefore by studying the surface current distributions at two orthogonal modes, formulation in resonant length in terms of slot and patch dimensions for non-suspended and suspended configurations, is proposed. The frequencies calculated using them closely agree with the simulated results. Further using proposed formulations, procedure to design similar slot cut CP CMSAs at different frequencies and on different substrates, is presented. At respective frequencies, it gives CP response with formation of small loop inside VSWR $=2$ circle. Thus, proposed formulation

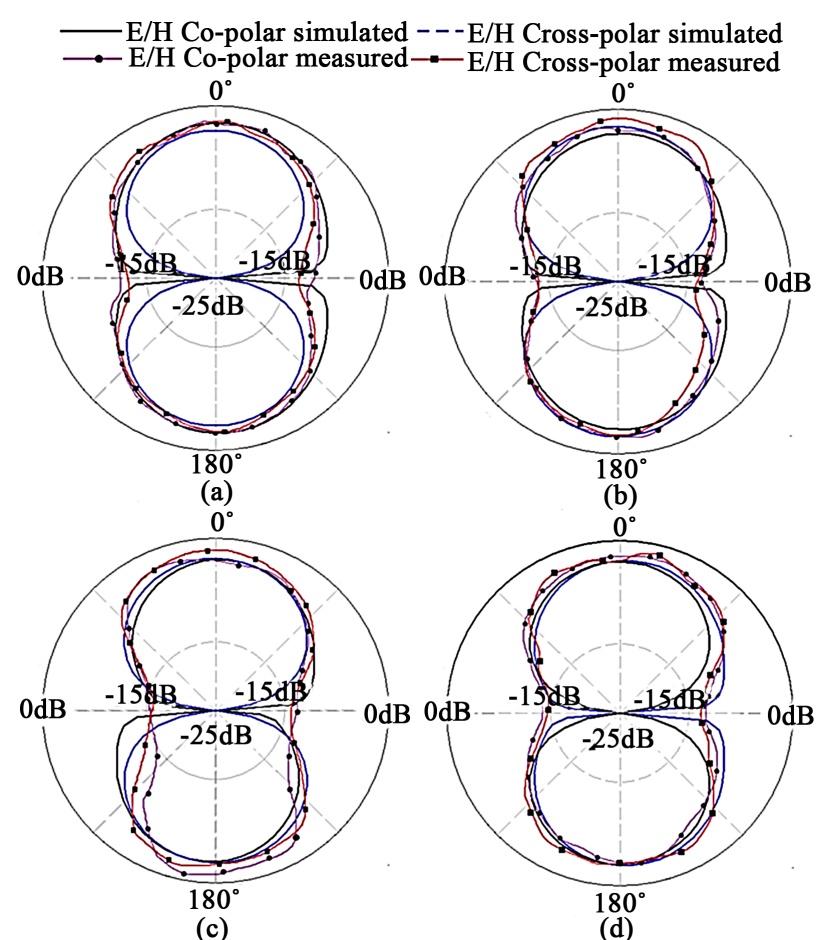

Figure 13. Radiation pattern at center frequency of AR BW at ((a), (b)) 1500 and ((c), (d)) $3000 \mathrm{MHz}$ for suspended slot cut CP CMSAs. (a) (b) $\mathrm{E}, \mathrm{f}=1500 \mathrm{MHz}$, (c) (d) $\mathrm{E}, \mathrm{f}=3000 \mathrm{MHz}$. 


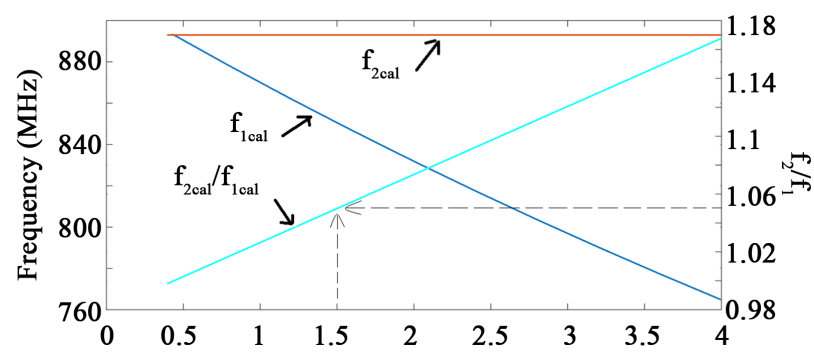

(a) width $(\mathrm{cm})$

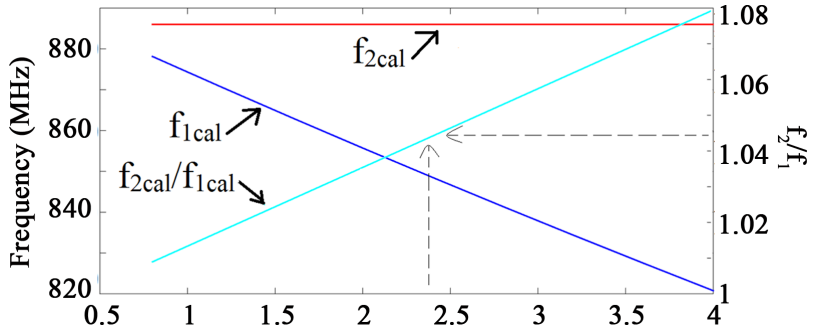

(b) width (cm)

Figure 14. Dual frequencies and their ratio plots for (a) non-suspended and (b) suspended CMSAs on RT-duroid substrate for $\mathrm{f}_{\mathrm{TM} 11}=900 \mathrm{MHz}$.

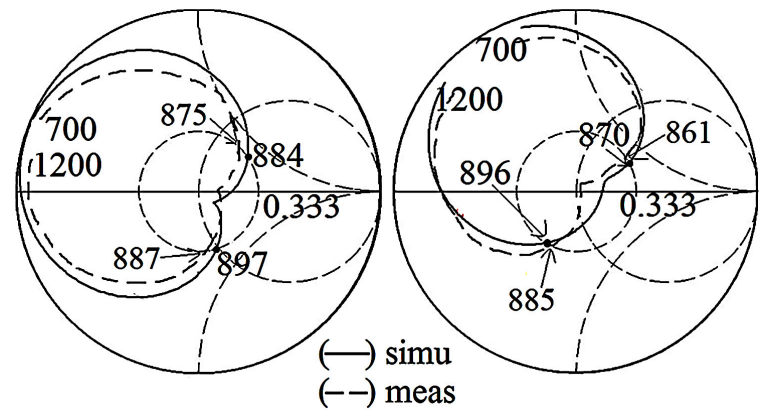

(a)

(b)

Figure 15. Input impedance plots for (a) non-suspended and (b) suspended CMSAs on RTduroid substrate for $\mathrm{f}_{\mathrm{TM} 11}=900 \mathrm{MHz}$.

and design procedure will be helpful for designing similar slot cut CP patch antennas. The proposed work can also provide some guideline to realize similar design procedure for other slot cut variations of CMSAs, rectangular MSA, equilateral triangular MSA and other modified patch shapes.

\section{References}

[1] Kumar, G. and Ray, K.P. (2003) Broadband Microstrip Antennas. 1st Edition, Artech House, Norwood.

[2] Garg, R., Bhartia, P., Bahl, I. and Ittipiboon, A. (2001) Microstrip Antenna Design Handbook. 1st Edition, Artech House, Norwood.

[3] Bhartia, B. and Bahl, I.J. (1980) Microstrip Antennas, USA.

[4] Nasimuddin, Chen, Z.N. and Quing, X. (2012) A Compact Circularly Polarized Cross-Shaped Slotted Microstrip Antenna. IEEE Transactions on Antennas and Propagation, 60, 1584-1588. http://dx.doi.org/10.1109/TAP.2011.2180334

[5] Yang, S.L.S., Lee, K.F. and Kishk, A.A. (2008) Design and Study of Wideband Single Feed Circularly Polarized Microstrip Antennas. Progress in Electronics Research, 80, 45-61. http://dx.doi.org/10.2528/PIER07110604

[6] Kasabegoudar, V.G. and Vinoy, K.J. (2009) Design and Study of Broadband Suspended Microstrip Antenna for Circular Polarization. Progress in Electronics Research, 90, 353-368. http://dx.doi.org/10.2528/PIER09012901

[7] Deng, J., Guo, L., Fan, T., Wu, Z., Hu, Y. and Yang, J. (2013) Wideband Circularly Polarized Suspended Patch An- 
tenna with Indented Edge and Gap-Coupled Feed. Progress in Electronics Research, 135, 151-159. http://dx.doi.org/10.2528/PIER12111601

[8] Sharma, P.C. and Gupta, K.C. (1983) Analysis and Optimized Design of Single Feed Circularly Polarized Microstrip Antennas. IEEE Trans Antennas and Propagation, 31, 949-955. http://dx.doi.org/10.1109/TAP.1983.1143162

[9] Lam, K. Y., Luk, K. M., Lee, K. F., Wong, H., and Bong, K. (2011) Small Circularly Polarized U-Slot Wideband Patch Antenna. IEEE Antennas and Wireless Propagation Letters, 10, 87-90.

[10] Khidre, A., Lee, K.F., Yang, F. and Eisherbeni, A. (2010) Wideband Circularly Polarized E-Shaped Patch Antenna for Wireless Applications. IEEE Antennas and Propagation Magazine, 52, 219-229. http://dx.doi.org/10.1109/MAP.2010.5687547

[11] Tong, K.F. and Wong, T.P. (2007) Circularly Polarized U-Slot Antenna. IEEE Transactions on Antennas and Propagation, 55, 2382-2385. http://dx.doi.org/10.1109/TAP.2007.901930

[12] IE3D 12.1, Zeland Software, Fremont, 2004. 